\title{
Mitochondria in skin health, aging, and disease
}

\author{
Annapoorna Sreedhar ${ }^{1}$, Leopoldo Aguilera-Aguirre ${ }^{1}$ and Keshav K. Singh ${ }^{2,3}$
}

\begin{abstract}
The skin is a high turnover organ, and its constant renewal depends on the rapid proliferation of its progenitor cells. The energy requirement for these metabolically active cells is met by mitochondrial respiration, an ATP generating process driven by a series of protein complexes collectively known as the electron transport chain (ETC) that is located on the inner membrane of the mitochondria. However, reactive oxygen species (ROS) like superoxide, singlet oxygen, peroxides are inevitably produced during respiration and disrupt macromolecular and cellular structures if not quenched by the antioxidant system. The oxidative damage caused by mitochondrial ROS production has been established as the molecular basis of multiple pathophysiological conditions, including aging and cancer. Not surprisingly, the mitochondria are the primary organelle affected during chronological and UV-induced skin aging, the phenotypic manifestations of which are the direct consequence of mitochondrial dysfunction. Also, deletions and other aberrations in the mitochondrial DNA (mtDNA) are frequent in photo-aged skin and skin cancer lesions. Recent studies have revealed a more innate role of the mitochondria in maintaining skin homeostasis and pigmentation, which are affected when the essential mitochondrial functions are impaired. Some common and rare skin disorders have a mitochondrial involvement and include dermal manifestations of primary mitochondrial diseases as well as congenital skin diseases caused by damaged mitochondria. With studies increasingly supporting the close association between mitochondria and skin health, its therapeutic targeting in the skin-either via an ATP production boost or free radical scavenging - has gained attention from clinicians and aestheticians alike. Numerous bioactive compounds have been identified that improve mitochondrial functions and have proved effective against aged and diseased skin. In this review, we discuss the essential role of mitochondria in regulating normal and abnormal skin physiology and the possibility of targeting this organelle in various skin disorders.
\end{abstract}

\section{Facts}

- Skin is the largest organ with high turnover rate in the human body.

- Mitochondria play a vital role in the skin.

- Mitochondrial dysfunction induces skin aging.

- Skin disorders manifest mitochondrial dysfunction.

- Targeting mitochondria may help rejuvenate skin.

Correspondence: Keshav K. Singh (kksingh@uab.edu)

YYuva Biosciences, 1500 1st Avenue N, Birmingham, AL 35203, USA

2Department of Genetics, University of Alabama at Birmingham, Birmingham, AL 35294, USA

Full list of author information is available at the end of the article

Edited by E. Candi

\section{Open Questions}

- How do mitochondria regulate skin aging?

- Is mitochondrial dysfunction a primary or a secondary cause of skin aging?

- Does targeting mitochondria prevent or slow down skin aging?

\section{Introduction}

The skin is the outer sheath of the body, which interfaces with the external environment. It is a complex structure made of several tissue layers, each with a distinct cellular composition. It has crucial functions like sensation, heat insulation and prevention of water loss, and acts as a physical barrier to pathogens. The upper layer of the skin is the epidermis, a stratified structure interspersed with hair follicles. The outer epidermal surface is made of 
cornified keratinocytes or corneocytes that form a dense cytoskeletal network of keratin filaments. At the same time, the basal layer harbors the epidermal stem and progenitor cells, which continuously regenerate the epidermis. The dermis lies underneath the epidermis and consists of dermal fibroblasts that produce collagen and elastin that form the extracellular matrix (ECM), as well as the melanocytes that produce the photo-protective pigment melanin ${ }^{1}$. With aging, both the epidermis and dermis undergo thinning and lose their regenerative capacity, which manifests as wrinkling, dryness, and mottling. Also, chronic exposure to environmental elements like ultraviolet (UV) radiations cause deleterious effects in the skin cells ${ }^{2}$. UVA and UVB radiation are not only associated with premature skin photo-aging but also various inflammatory reactions and skin cancer ${ }^{3}$.

The skin is a high turnover organ, with a continuously regenerating epidermis. The epidermal progenitors are thus highly proliferative and metabolically active and rely on adenosine triphosphate (ATP) for their energy requirements. ATP is mainly produced through oxidative phosphorylation (OXPHOS) in the mitochondrion, which is the bioenergetics center of the eukaryotic cell ${ }^{4}$. This double membrane-bound organelle is involved in essential functions like energy production, fatty acid oxidation (FAO), heme and steroid biosynthesis, apoptosis, and calcium signaling. Mitochondrial respiration utilizes a series of protein complexes located at the inner membrane, collectively known as the electron transport chain (ETC). These complexes sequentially transport electrons and translocate protons to the intermembrane space creating a proton gradient, which is utilized to generate $\mathrm{ATP}^{5}$ (Fig. 1). The natural by-products of OXPHOS include reactive oxygen species (ROS) such as superoxide anion, singlet oxygen and peroxides, which can disrupt macromolecular and cellular structures. Although ROS production can be triggered by external stimuli such as ionizing radiations and carcinogens, the generation of endogenous ROS is highest in the mitochondria ${ }^{6}$. The oxidative damage caused by mitochondrial ROS production is an essential molecular basis of various pathophysiological conditions, including aging and cancer ${ }^{7}$.

Mitochondria are unique organelles that possess a maternally inherited autonomous genome. The mitochondrial DNA (mtDNA) encodes for 13 OXPHOS proteins, in addition to 22 tRNAs and two rRNAs ${ }^{8}$ (Fig. 2). Due to their physical proximity to the ETC and therefore, ROS, mtDNA is highly susceptible to mutations, which invariably disrupt OXPHOS, thus triggering a vicious cycle of increased ROS production and mtDNA damage ${ }^{9}$. Somatic mutations in mtDNA are often observed in various diseases, including cancer. Besides, mtDNA exists as multiple copies, and studies have associated abnormal copy numbers with different pathophysiological states ${ }^{10}$.

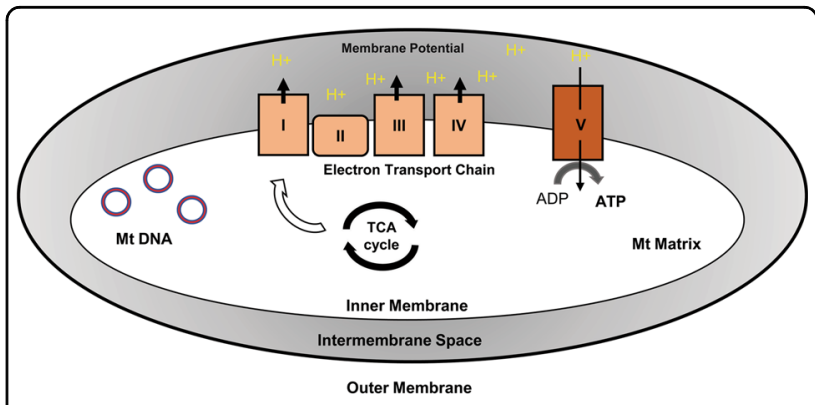

Fig. 1 Mitochondria in skin health. Mitochondrion is a doublemembrane organelle found in the cytoplasm of almost all eukaryotic cells. It is the site of cellular respiration and most ATP production. Mitochondrial respiration utilizes a series of protein complexes: complex I (NADH dehydrogenase), complex II (succinate dehydrogenase), complex III (cytochrome c reductase) and complex IV (cytochrome c oxidase) located in the inner mitochondrial membrane. These complexes sequentially translocate electrons $\left(\mathrm{e}^{-}\right)$to create a proton gradient $\left(\mathrm{H}^{+}\right)$. Complex $V$ (ATP synthase) then makes use of this gradient to phosphorylate ADP to ATP.

Some mtDNA mutations or single nucleotide polymorphisms (SNPs) can persist in a population, giving rise to haplogroups with distinct geographical distribution; at present, around 27 major mitochondrial haplogroups are known, and many have been correlated with a higher risk of cancers and other diseases, including those of the skin $^{11}$.

Recent studies have established an essential role of the mitochondria in skin homeostasis, as well as disorders such as cancer, inflammatory conditions, and rare inherited diseases. The mechanistic basis of mitochondrial dysfunction in the skin and other organs, is primarily ROS production and oxidative stress, although other mitochondrial pathways have also been linked with certain skin disorders (Fig. 3). In this review, the essential role of mitochondria in regulating normal skin physiology, as well as in healthy/premature skin aging and skin cancers, have been discussed. Also, the therapeutic targeting of mitochondria in various skin disorders has been considered.

\section{Role of mitochondria in skin homeostasis Mitochondria-dependent epidermal differentiation}

The epidermis is a self-renewing layer that depends on the subsequent differentiation and migration of the proliferative basal keratinocytes to the cornified keratinocytes in the outermost squamous layer ${ }^{12}$. Studies increasingly show that mitochondrial ROS generation plays a regulatory role in the differentiation of various cell lineages via several downstream transcriptional factors like C/EBP, Notch, and $\beta$-catenin, and increased ROS levels promote differentiation of murine embryonic stem cells (ESCs), induced pluripotent stem cells (iPSCs), as well as tissuespecific multipotent epithelial stem cells ${ }^{13}$. Hamanaka 


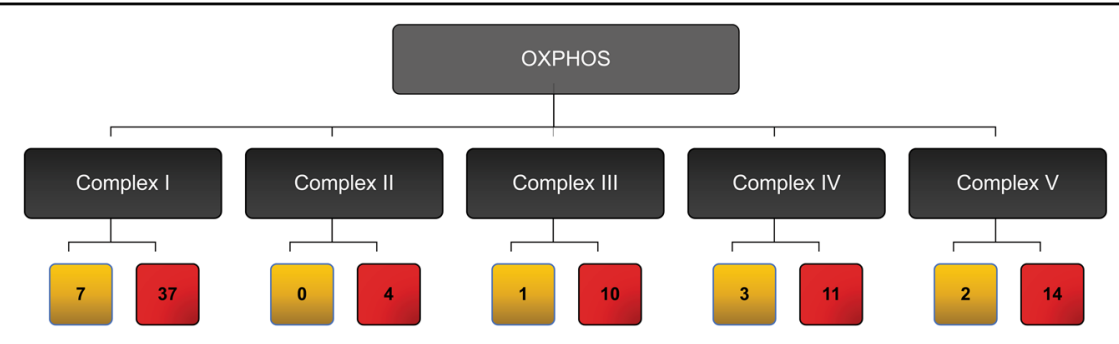

Mt DNA encoded

Nuclear DNA encoded

Fig. 2 Mitochondrial OXPHOS subunit composition. Schematic representation of mammalian respiratory chain complex. The ETC consists of five complexes, with subunits encoded by both mtDNA (yellow) and nuclear DNA (red). The human mtDNA genome encodes only 13 OXPHOS proteins, while the nuclear DNA encodes the remaining $80 \%$ of the OXPHOS proteins.

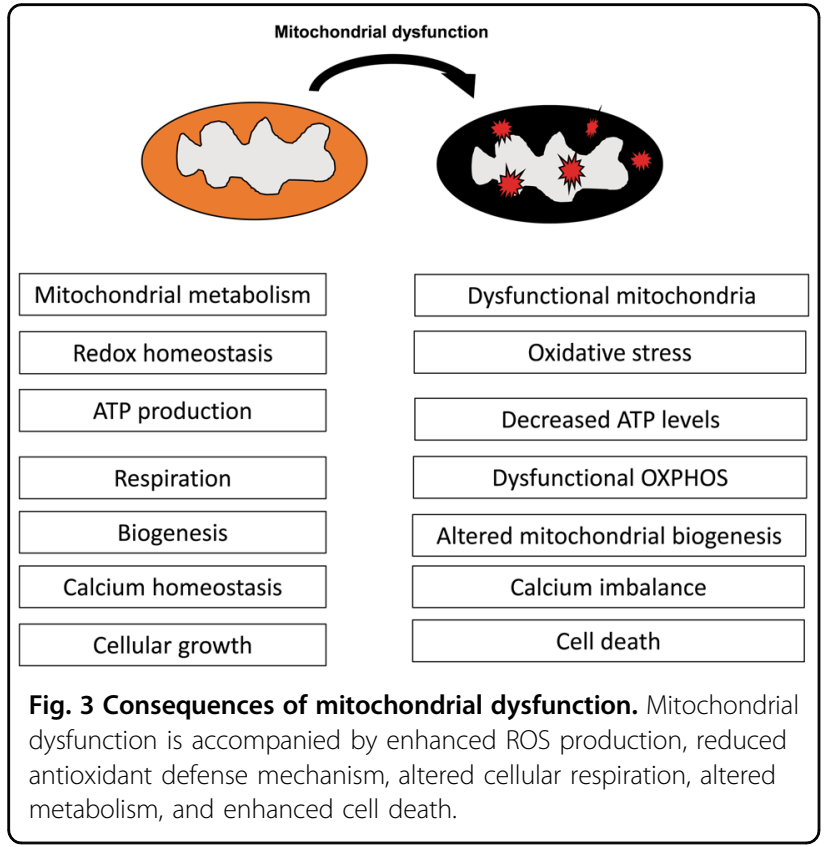

et al. ${ }^{14}$ found that conditional mitochondrial transcription factor A (TFAM)-knockout mice had a high neonatal mortality rate due to impaired epidermal barrier function and lacked normal fur. Also, primary keratinocytes isolated from these mice failed to differentiate in vitro. Since TFAM drives mtDNA replication and cells lacking TFAM have defective OXPHOS and low ROS levels, it is reasonable to surmise that mitochondrial respiration and ROS production are essential for keratinocyte differentiation. Indeed, antioxidant treatment of wild-type keratinocytes blocked their differentiation, while exogenous administration of $\mathrm{H}_{2} \mathrm{O}_{2}$ restored the same in TFAM $^{-1}$ - keratinocytes. Further mechanistic analysis revealed that mROS promoted epidermal differentiation by activating the Notch and $\beta$-catenin signaling cascades. Besides, low levels of $\mathrm{H}_{2} \mathrm{O}_{2}$ generated in the skin fibroblasts exposed to physiological doses of UVB rays activated the keratinocyte growth factor receptor and promoted keratinization.

Mitochondrial respiration also requires $\mathrm{Ca}^{2+}$ uptake, and a $\mathrm{Ca}^{2+}$ gradient has been observed in the epidermis in vivo ${ }^{15}$. Keratinocyte differentiation in vitro is enhanced by increasing $\mathrm{Ca}^{2+}$ levels, whereas the opposite effects are seen upon inhibiting mitochondrial calcium uptake ${ }^{16}$. In a recent study, Bhadhuri et al. ${ }^{17}$ used the gene networkbased bioinformatics approach to screen for hub proteins involved in epidermal differentiation and identified the mitochondrial proteins MPZL3 and FDXR. Functional analyses further showed that the proteins co-localized proximally in the keratinocyte mitochondria and promoted their differentiation by inducing ROS generation. Interestingly, depletion of the myelin protein zero-like 3 (MPZL3) is associated with dermatitis and alopecia in mice, and the ETC enzyme ferredoxin reductase (FDXR) sensitizes various human epithelial cell lines to ROSinduced apoptosis. Allombert-Blasie et al. ${ }^{18}$ showed that the terminal differentiation of keratinocytes was accompanied by the activation of the mitochondrial caspasedependent apoptotic pathway

\section{The melatonin-mitochondria axis in the skin}

Melatonin (N1-acetyl-5-methoxytryptamine) is a neurohormone secreted by the pineal gland that regulates the circadian rhythm in mammals. It is also synthesized in other organs like the retina, cochlea, lungs, liver, kidney, immune system, and the skin, and its levels are tightly regulated by both hepatic and epidermal metabolism ${ }^{19}$. It has a pivotal photo-protective role in the cutaneous tissues, in addition to regulating wound healing, pigmentation, inflammation, and immune responses. Melatonin and its metabolites directly act on skin cells expressing the 
G protein-coupled melatonin type (MT) 1 and 2 receptors following UV or X-ray exposure, and attenuate macromolecule damage by scavenging the radiation-induced free radicals ${ }^{20}$. Pharmacological doses of melatonin protect keratinocytes and melanocytes from UVB-induced damage in vitro $^{21}$. Full-thickness human skin histocultured ex vivo showed significantly lower levels of the DNA damage marker 8-hydroxy-2'-deoxyguanosine (8OHdG) following UV exposure when pre-treated with melatonin $^{22}$. Apart from direct scavenging of ROS like $\mathrm{H}_{2} \mathrm{O}_{2}$, singlet oxygen, and $\mathrm{NO}^{-}$, melatonin and its derivatives also protect the skin cells from radiation damage by increasing the activity of antioxidant enzymes ${ }^{22,23}$. Interestingly, high levels of endogenous melatonin have been detected in human and rodent skin cells, including melanocytes, keratinocytes, and epithelial cells, in addition to the enzymes involved in melatonin synthesis and metabolism $^{19}$.

The potent radio-protective and antioxidant role of melatonin in the skin raises the possibility of mitochondrial involvement. Indeed, studies have increasingly pointed to a "symbiotic" relationship between melatonin and epidermal mitochondria, with the latter serving as the site of cutaneous melatonin biosynthesis and metabolism. In contrast, melatonin has regulatory effects on the mitochondrial ETC and antioxidant machinery. Melatonin is metabolized in the mitochondria via the cytochrome c and $\mathrm{H} 2 \mathrm{O} 2$-dependent kynurenic pathway, and the cytochrome P450-dependent indolic pathway ${ }^{19,24}$. The primary metabolites of melatonin include N1-acetylN2-formyl-5-methoxykynuramine (AFMK), 6-hydroxylmelatonin, and $\mathrm{N}$-acetylserotonin, which accumulate in the epidermis following UVB exposure in a dosedependent manner ${ }^{19}$. A melatonin biosynthetic machinery may also be present in the mitochondria since oocyte mitochondria can synthesize melatonin from serotonin in a cell-free system ${ }^{25}$. It remains to be elucidated whether cutaneous mitochondria also have a similar function. Melatonin can directly increase the electron flux through the respiratory chain and enhance ATP production by donating electrons. One study showed that 6hydroxymelatonin promoted electron transfer from complex III to complex IV (cytochrome c oxidase), which may help restore the age-dependent decline in mitochondrial bioenergetics. Besides, melatonin mediates the reduction of cytochrome $c$ and augments $\mathrm{H}_{2} \mathrm{O}_{2}$ scavenging ${ }^{26}$.

The intracellular $\mathrm{Ca}^{2+}$ homeostasis-regulating protein complex calmodulin also binds to melatonin, indicating the role of the latter in mitochondrial functions like autophagy and apoptosis as well ${ }^{27}$. One study showed that topical application of melatonin improved the barrier function of the epidermis by increasing keratinocyte proliferation $^{28}$. It can also accelerate wound healing and augment the effects of antimicrobials ${ }^{29}$. Given that ROS production also plays a role in tissue repair and regeneration $^{30}$, the melatonin-mitochondrial axis may also be the driver of epidermal wound healing.

\section{Mitochondrial role in skin aging}

In addition to the inevitable time-induced changes, the skin is also highly susceptible to photo-aging due to chronic exposure to solar UVA and UVB radiations ${ }^{2}$. Aging affects the protective role of the skin against physicochemical and biological attacks, as well as its thermoregulatory, sensory, immunological, and hormonal functions. The structural and functional manifestations of cutaneous aging are premature and more severe in the photo-aged compared to the chronologically aged skin. Furthermore, since photoaging is a cumulative process, it is more pronounced among older individuals who have been regularly exposed to the sun for long periods. Both intrinsic and environmental factors affect the epidermal and dermal layers of the skin. Histologically, chronological aging is characterized by significant epidermal thinning, which manifests as dryness and wrinkles ${ }^{1}$.

In contrast, photo-aged skin has a thick, leathery appearance with deeper wrinkles and uneven pigmentation $^{31}$. Several studies have directly or indirectly linked mitochondrial dysfunction to both chronological and photo-aging of the $\operatorname{skin}^{32}$. At the molecular levels, aged skin is characterized by damaged mitochondria, mtDNA deletions, high ROS levels, and oxidative stress in both the dermal and epidermal layers (Fig. 4).

\section{Chronological skin aging}

Mutations and deletions in mtDNA are known to accumulate in post-replicative tissues with aging, which is accompanied by a steady decline in mitochondrial function $^{33}$, increased ROS production, and loss of mitochondrial membrane potential (MMP), followed by increased mitophagy, and apoptosis. A 4977 base pairs extended region of mtDNA, including the genes encoding complexes I, IV, and V of the respiratory chain, is frequently deleted in the aged human skin". This "common deletion" is more evident in the skin of individuals older than 70 years and increases with sun exposure ${ }^{34}$. Kaneko et al. ${ }^{35}$ compared the neck skin samples of German and Japanese women and found a general increase in the frequency of the common deletion with age, and in sunexposed versus photo-protected skin. Besides, a strong positive correlation was observed between the density of common deletion and skin wrinkles among the Japanese women, while the higher frequency of common deletion correlated with fewer pigmented lesions among the German women. The latter also showed a slightly higher amount of the common deletion compared to Japanese women, which may be due to intrinsic genetic variations, 

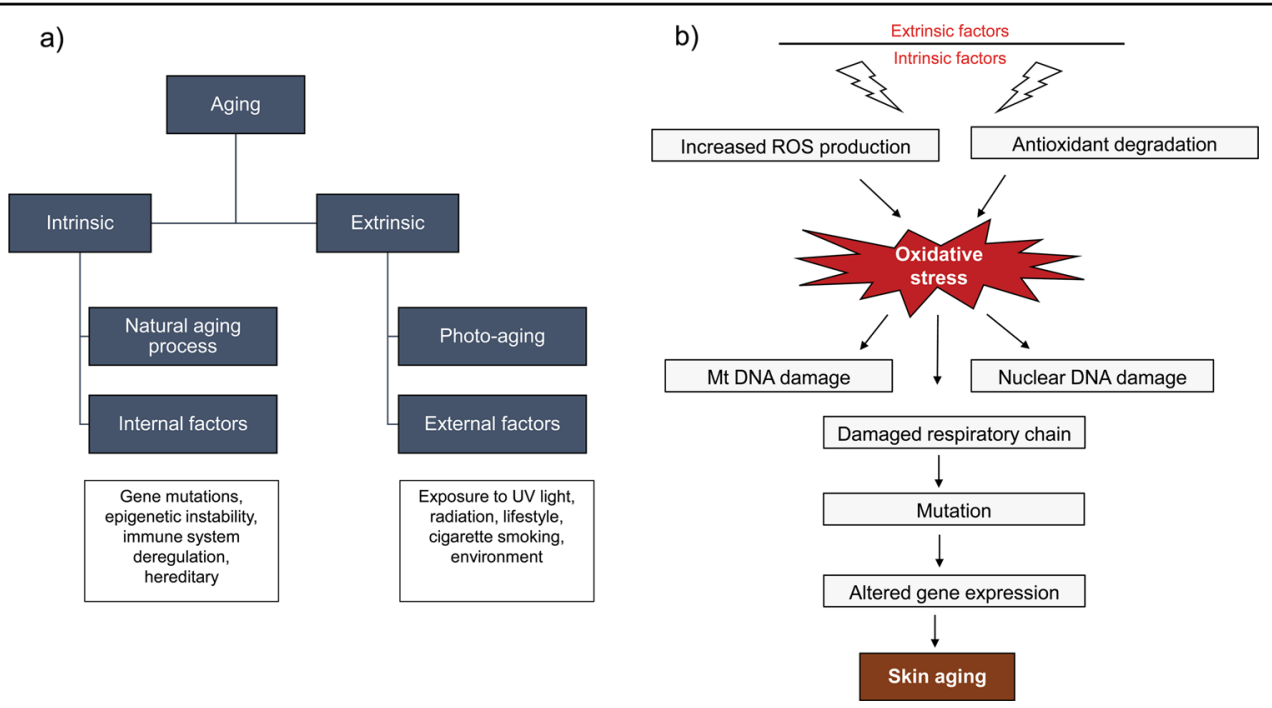

Fig. 4 Mitochondria in skin aging. a Skin aging categories: Intrinsic (internal) aging and extrinsic (external) aging. b Skin aging is characterized by loss of membrane potential, elevated levels of ROS, DNA damage leading to nuclear and mitochondrial gene mutations, respiratory chain defect due to enzyme alterations, altered cellular regulation, and disease progression.

or different cultural attitudes towards sun exposure. In a recent study, Singh et al. ${ }^{36}$ established an inducible mtDNA-depletion mouse model with a dominantnegative mutation in the mtDNA polymerase POLG1. The mice had ubiquitous mtDNA depletion and correspondingly reduced OXPHOS. The phenotypic manifestations included extensive skin wrinkling and fur loss, which in turn were associated with epidermal hyperplasia and hyperkeratosis. The transgenic mice had increased levels of matrix metalloproteinases that are often increased during skin aging. Interestingly, turning off the mutant POLG1 expression restored the cutaneous pathologies to the wild-type level. This study is the first to prove that mtDNA depletion is the underlying cause of skin aging and that restoring mitochondrial functions can restore skin youthfulness.

The age-dependent accumulation of ROS in the keratinocytes, and the concomitant loss of MMP, results in a metabolic shift from OXPHOS to the anaerobic glycolysis. Prahl et al. ${ }^{37}$ isolated keratinocytes from skin biopsies of old and young donors and found a distinctly glycolytic phenotype of the older keratinocytes, and addition of the ETC component coenzyme (Co) Q10 restored mitochondrial metabolism in the aged cells. Consistent with this, an age-related decline in complex II (succinate oxidoreductase) activity has also been observed in aged human skin fibroblasts ${ }^{38}$. Damaged mitochondria are cleared away by a highly conserved pathway called mitophagy, or the selective autophagy of mitochondria ${ }^{39}$. Mitophagy levels increase considerably after cellular stress or damage, and homeostasis between mitochondrial biogenesis and mitophagy is vital for a healthy mitochondria pool. Aymard et al. ${ }^{40}$ demonstrated a critical role of autophagy and mitophagy in keratinocyte differentiation, which also raises the possibility of mitochondrial fragmentation in aged keratinocytes owing to the increase in ROS levels with aging and elevated mitochondrial fission in response to oxidative stress. Recently, Mellem et al. ${ }^{41}$ studied the mitochondrial network in young and old human skin for the first time in vivo and found significantly fewer mitochondrial clusters in the keratinocytes. A highly connected physical network of mitochondria in the epidermal cells of the younger compared to the older skin. The latter had a significantly fragmented mitochondrial network, indicating poor recycling and excessive mitophagy. The similarities in mitochondrial dynamics in normal differentiation and aging could either be due to common pathways that are dysregulated during aging or simply due to the agingrelated reduced epidermal turnover ${ }^{42}$.

Coenzyme Q (CoQ10) is a lipophilic isoprenylated quinone that acts as an electron shuttle between complex I/II and complex III of the ETC, and as a ROS scavenger that protects against membrane lipid oxidation ${ }^{43}$. Both the antioxidant and bioenergetic roles of CoQ10 are closely associated with skin aging and other disorders. CoQ10 levels are 10-fold higher in the epidermis compared to the dermis and decrease significantly with age. Reduced CoQ10 content in aged dermal fibroblasts is associated with lower activity of the complexes I/III and II/III, membrane depolarization, and generation of superoxide anions ${ }^{44}$. Furthermore, several studies have shown that topical application of CoQ10 on photo-aged skin ameliorates the phenotypic signs of aging and 
restores mitochondrial function ${ }^{45}$. The age-related glycolytic shift reported by Prahl et al. ${ }^{37}$ in the human keratinocytes was also associated with impaired CoQ10 function and was reversed by its exogenous application. CoQ10 is synthesized de novo by the mevalonate pathway and can be inhibited by 3-hydroxy-3-methyl-glutarylcoenzyme A (HMG-CoA) reductase inhibitors like statins. Marcheggiani et al. ${ }^{46}$ recently showed that statin mediated CoQ10 inhibition in human dermal fibroblasts triggered oxidative stress and mitochondrial dysfunction, and lead to premature "aging" of the cells in vitro.

\section{Photo-aging}

Chronic UV exposure induces nuclear and mitochondrial DNA damage and oxidative stress in the skin cells, which can progress to photo-aging and or skin cancer. UVB mainly acts on the epidermal keratinocytes and melanocytes, while UVA can penetrate more deeply into the dermis ${ }^{47}$. A cardinal marker of photo-aging, which is relatively less obvious in chronological aging is large-scale mtDNA deletion. Berneberg et al. ${ }^{48}$ reported a 10 -fold higher rate of a $\sim 5000 \mathrm{bp}$ deletion in the mtDNA of photo-aged compared to sun-protected skin cells. In a later study, the same group ${ }^{49}$ found that repeated exposure of the normally unexposed buttock skin to UVA increased the frequency of mtDNA deletions in the dermal cells by $40 \%$ compared to the non-irradiated skin.

Interestingly, these UV-induced deletions can persist over many years and can increase by $30-40$ folds even in the absence of further irradiation ${ }^{47,48}$. UVA/B exposure, however, is not homogenous across the entire epidermis, since some parts of the body are more frequently exposed to the sun than others. Krishnan et al. ${ }^{50}$ observed a significantly higher frequency of a $3895 \mathrm{bp}$ mtDNA deletion block in habitually exposed areas of the skin compared to the less exposed regions. In a recent study by Powers et al. ${ }^{51}$ an unusually high rate of the common mtDNA deletion (4977 bp) and a 3895-bp deletion was observed in the sun-exposed skin biopsies of elderly individuals that were diagnosed negative for any skin cancer.

Repeated exposure to sub-lethal doses of UVA resulted in mtDNA deletions in primary human keratinocytes ${ }^{52}$ and dermal fibroblasts, which was accompanied by decreased mitochondrial function, increased activity of collagen-degrading enzymes ${ }^{53}$ and oxidative stress, which further exacerbated the mitochondrial damage $\mathrm{e}^{54}$. Schroeder et al..$^{55}$ generated mtDNA-depleted human skin fibroblast lines. They found that in addition to aberrantly high oxidative stress, these cells showed the typical molecular features of photo-aged skin, such as increased levels of collagen degrading metalloproteases (MMPs) and downregulation of the genes involved in collagen biosynthesis ${ }^{45}$, which translates to the extensive wrinkling of photo-aged $\operatorname{skin}^{31}$. This is consistent with the hypothesis of altered collagen metabolism in photo-aging, first proposed by Fisher et al. ${ }^{46}$. Furthermore, skin fibroblasts isolated from patients with Kearns-Sayre syndrome (KSS), a rare inherited disorder characterized by extensive mtDNA deletions, showed higher ROS levels and increased activity of photo-aging-related genes ${ }^{56}$. Oyewole et al. ${ }^{57}$ compared the protective effects of mitochondriaspecific antioxidants and cellular antioxidants (resveratrol and curcumin) against UVA-induced mtDNA damage in human dermal fibroblasts. They detected significantly higher photo-protection by the former. These findings collectively indicate that UV-induced photo-aging is directly linked to mtDNA depletion and that the phenotypic features of photo-aged skin are the result of mitochondrial dysfunction and ECM degradation.

In addition to the UV radiation, infrared (IR) rays have also been associated with photo-aging of human skin. Schieke et al. ${ }^{58}$ showed that exposure to IR dysregulated the expression of metalloproteases in primary human fibroblasts. Similar changes were observed in situ in human skin exposed to $\mathrm{IR}^{54}$, while chronic exposure to IR also resulted in the typical clinical signs of photo-aging in animal models ${ }^{59}$. Schauen et al. ${ }^{60}$ detected a significant increase in the intra-mitochondrial levels of ROS in primary human skin fibroblasts exposed to IR, indicating a direct role of the mitochondria in IR-induced photoaging. Consistent with this, treatment of IR-exposed human fibroblasts with either an antioxidant or an ETC inhibitor reversed the signs of photo-aging ${ }^{61}$. The underlying mechanism of IR-induced mitochondrial damage was elucidated by Karu et al. ${ }^{62}$ who showed that the IR rays are absorbed by the copper atoms of ETC complex IV, leading to disturbed electron flow and 'leakage,' and increased ROS production.

Mitochondrial perturbations, such as mtDNA mutations/deletions, loss of MMP, and the uncoupling of the OXPHOS machinery induces mito-nuclear retrograde signaling. The effect of retrograde signaling is a global alteration in nuclear gene expression, which leads to metabolic shift, apoptosis, and mitophagy. In recent years, retrograde mitochondrial signaling has been directly linked with various pathological states. In the context of photo-aging, retrograde mitochondrial signaling alters the expression of nuclear genes involved in collagen biosynthesis and degradation, inflammation, and neovascularization, all of which contribute to the appearance of the photo-aged skin $^{61}$. The presence of oxidized proteins in the upper dermal layers of photo-damaged skin lends support to this hypothesis ${ }^{63}$.

While chronological aging is associated with a $10-20 \%$ decrease in melanin production per decade, chronic UV exposure increases the amount of this photo-protective pigment, resulting in the typical "mottled" appearance of photo-aged $\operatorname{skin}^{64}$. Melanocytes are highly susceptible to 
oxidative damage since melanogenesis is pro-oxidative and have higher basal levels of ROS compared to other cell types. Melanocytes from UV-exposed skin show an increase in ROS and melanin levels compared to the cells isolated from normal skin. While endogenous melanin is known to protect melanocytes from photo-oxidative damage by absorbing the UV rays, chronic UV exposure can lead to melanin oxidation, which in turn increases ROS levels and further aggravates the oxidative damage. There is evidence indicating that the melatonin-regulated cutaneous circadian clock also controls melanogenesis in the human epidermis and cultured fibroblasts ${ }^{65}$.

\section{Mitochondrial role in skin cancer}

Skin cancer is the most common malignancy affecting humans and accounts for $40 \%$ of all cancer cases worldwide. It is classified into the non-melanoma skin cancers (NMSCs) that include basal-cell carcinoma (BCC) and squamous cell carcinoma (SCC), and the melanocytederived melanomas. Although the latter comprise only $5 \%$ of all skin cancer cases, they are associated with a significant mortality rate of $\sim 25 \%$ as well as metastasis risk ${ }^{66}$. Skin cancer risk is linked to UV radiation, and the incidence of all skin cancer types have significantly increased in the last 2-3 decades due to increased exposure to UV rays, both due to the thinning ozone layer and frequent use of tanning beds. The risk of developing skin cancer, especially malignant melanoma, is also higher in the Caucasian population on account of their lower cutaneous melanin content ${ }^{67}$. Mitochondria have been implicated in almost all human cancers, primarily on account of their role in ROS generation and apoptosis. Congenital and somatic mutations in mtDNA have been detected in most tumors, including those affecting the $\operatorname{skin}^{68-70}$, and tumorigenic stimuli such as radiation and carcinogens are known to damage the mtDNA directly ${ }^{71-75}$. Furthermore, mounting evidence suggests that mitochondria are involved in the interplay between oxidative stress, hypoxia $^{76-81}$, and metabolic reprogramming of tumor cells ${ }^{82-86}$.

\section{Mitochondrial involvement in other skin conditions}

In addition to skin aging and cancer, mitochondrial dysfunction has also been associated with various common and rare skin disorders that can be broadly classified into three types: dermal manifestations of primary mitochondriopathies, skin diseases due to mitochondrial dysfunction, and cutaneous manifestations of genetic diseases that affect mitochondria. Clinically, these disorders can be present as hair abnormalities, inflammation, rashes, hypo- and hyperpigmentation, and acrocyanosis (i.e., blueness of extremities). The underlying molecular mechanisms have been identified for most, and mainly involve dysfunctional OXPHOS and high ROS generation, mutations in mtDNA, an imbalance between mitochondrial biogenesis and mitophagy, and aberrant expression levels of nuclear-encoded mitochondrial proteins. In addition, dysregulation in other mitochondrial metabolic pathways and structural proteins have also been implicated. The entire gamut of skin diseases with direct or indirect mitochondrial involvement has been summarized in a recent review by Feichtlinger et al. ${ }^{87}$ (Fig. 5 and Table 1).

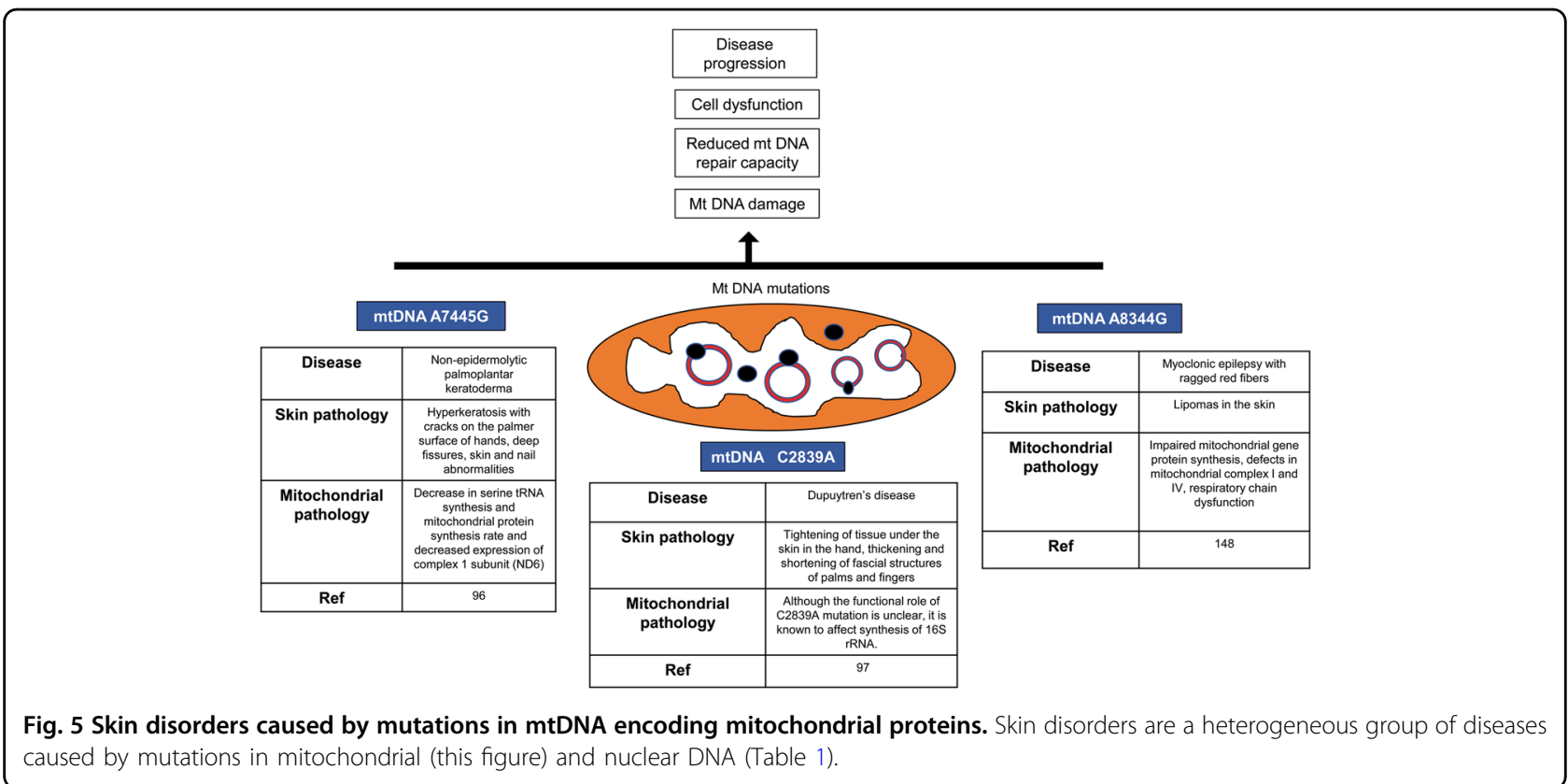


Table 1 Skin disorders caused by mutations in nuclear DNA affecting mitochondrial functions.

\begin{tabular}{ll}
\hline Gene & Skin pathology \\
\hline Ataxia-Telangiectasia Mutated (ATM) & $\begin{array}{l}\text { Telangiectasia, progeric and sclerodermatous } \\
\text { skin changes }\end{array}$
\end{tabular}

Biotinidase (BTD)

Mitochondrial chaperone (BCS1L)

Cytochrome c oxidase 7B (COX7B)

Coproporphyrinogen oxidase (CPOX)

Comparative gene identification-58 (CGI-58)

DNA excision repair protein ERCC6 (ERCC6)

Fanconi anemia complementation group A (FANCA)

Ferrochelatase (FECH)

Human HRas (HRAS) or transforming protein p21

Halocytochrome c-type heme lyase synthase (HCCS)

Isocitrate dehydrogenase (IDH1; IDH2)

Protoporphyrinogen oxidase (PPOX)

Lamin A/C (LMNA)

Menkes' protein (MNK)

Plectin 1 (PLEC1)

Pyrroline-5-carboxylate reductase 1 (PYCR1)

ATP-dependent DNA helicase Q4 (RECQL4)
Hypotonia, skin rashes, hair loss

Hypertrichosis, abnormal hair growth, brittle hair

Microphthalmia with linear skin lesions, aplastic skin, hyperpigmented areas on the skin, skin defects, linear areas of erythematous skin hypoplasia

Photosensitivity, skin lesions, jaundice

Congenital ichthyosiform erythroderma, abnormal scaling of the skin with underlying redness.

Freckled skin, scarring, pigmentation, atrophy, anhidrosis, decreased subcutaneous adipose tissue

Pigmentary changes, short stature, hyper pigmented skin, absence of left-hand thumb

Light-sensitive dermatitis, photosensitivity leading to skin thickening and areas of hyperkeratosis, skin itching and burning, erythema, edema, mild scarring

Loose redundant skin, coarse facial features, dark skin pigmentation, papillomas, abnormal thickening and darkening of skin, deep palma creases

Skin defects limited to the face and neck, aplastic skin, appearance of skin lesions, hyperpigmented areas of the skin

Enchondromata, small red scratches or bumps on the skin, hemangiomata

Skin photosensitivity, skin hyperpigmentation and hypertrichosis

Early loss of hair, patchy skin coloring, loss of fatty tissue under the skin, particularly affecting the limbs

Hypopigmentation, loose and sagging skin, coarse hair, loose joints

Skin blistering at birth or shortly thereafter, progressive muscle weakness, deformities of the nails, palmoplantar hyperkeratosis, and alopecia

Loose skin, loss of bone density

Skin rashes, hair loss, skin lesions, poikiloderma threadlike red lines or patterns on the skin, atrophy, sun hypersensitivity, ulcers
Mitochondrial pathology

Ref.

Mitochondrial abnormalities, elevated ROS, increased aberrant mitochondria, respiratory enzyme deficiency, defective mitochondrial respiration, decreased mitophagy.

Defective biotin metabolism, carboxylase and 99,120-122 biotinidase deficiency, ATP depletion, defective TCA cycle.

Mitochondrial complex III deficiency, defective mitochondrial respiration, changes in mitochondrial morphology, mitochondrial encephalopathy, multiple organ failure

OXPHOS defects, mitochondrial respiration deficiency, CNS defects, developmental

deficiencies

Decreased activity of CPOX, mitochondrial abnormalities, acute ataxia and iron-sulfur assembly defects.

Abnormal accumulation of intracellular lipid droplets in nonadipose tissues, dysregulated lipid homeostasis, myopathy, impaired fatty acid oxidation, increased mitochondrial size, reduced mtDNA content

Increased ROS, accumulation of damaged mitochondria, defective mitophagy, incompetent DNA repair system, altered cellular homeostasis.

Reduced electron transfer between respiratory complex I-III, reduced ATP/AMP ratio, deficiency of ROS detoxification enzymes.

Reduced activity of FECH, mitochondrial abnormalities, iron-sulfur assembly defects

Inactive HRAS protein, uncontrolled cell division, altered metabolism, mitochondrial dysfunction, defective OXPHOS

HCCS deficiency, enhanced cell death, reduced OXPHOS efficiency

nactive IDH enzyme, decreased aketoglutarate levels and increased accumulation of oncometabolite 2hydroxyglutarate, increased oxidative stress, dysfunctional TCA cycle

Reduced activity of enzyme PPOX, mitochondrial abnormalities, iron-sulfur assembly defects

Swollen mitochondria with loss of cristae, reduced mitochondrial function, decreased ATP generation, altered metabolism,

Reduced activity of MNK, reduced heme synthesis, diminished complex IV activity, dysfunctional OXPHOS

Aberrant plectin expression, reduced skeletal muscle function and network, increased skin fragility, muscular weakness, muscular dystrophy, myopathy, structural and functional alterations of mitochondria, reduced complex $I$, IV and $V$ activity, diminished OXPHOS

Altered mitochondrial morphology, increased oxidative stress, increased cell death

Altered mitochondrial integrity and mitochondrial bioenergetics, loss of mitochondrial reserve capacity, accumulation of damaged mtDNA
105,117-119

$123-125$

126

$100,127-129$

130,131

132,133 
Table 1 continued

\begin{tabular}{|c|c|c|c|}
\hline Gene & Skin pathology & Mitochondrial pathology & Ref. \\
\hline $\begin{array}{l}\text { RNA component of mitochondrial RNA processing } \\
\text { endoribonuclease (RMRP) }\end{array}$ & Hypoplastic hair & $\begin{array}{l}\text { Defective mitochondrial RNA-processing } \\
\text { endoribonuclease }\end{array}$ & 145 \\
\hline $\begin{array}{l}\text { SAM domain and HD domain-containing protein } 1 \\
\text { (SAMHD1) }\end{array}$ & $\begin{array}{l}\text { Skin rash, bluish or purple coloring of the } \\
\text { hands and feet, photosensitivity, purpura, } \\
\text { petechia, jaundice }\end{array}$ & $\begin{array}{l}\text { Replication defects, defective mitochondria } \\
\text { DNA synthesis, altered dNTP pool, complex I } \\
\text { deficiency }\end{array}$ & 146 \\
\hline Pemphigus vulgaris (PV) caused by MtABs & $\begin{array}{l}\text { Skin erosions or blistering formations on } \\
\text { the skin }\end{array}$ & $\begin{array}{l}\text { Increased ROS, altered mitochondrial } \\
\text { membrane potential, proton leakage, } \\
\text { OXPHOS defect }\end{array}$ & 110 \\
\hline $\begin{array}{l}\text { Sytemic lupus erythematosus (SLE) caused } \\
\text { by MtABs }\end{array}$ & $\begin{array}{l}\text { Skin inflammation, red rash, swollen joins, } \\
\text { hair loss }\end{array}$ & $\begin{array}{l}\text { Mitochondrial dysfunction in T cells / } \\
\text { lymphocytes, elevated ROS, hyperpolarized } \\
\text { mitochondria, ATP depletion }\end{array}$ & 69,108 \\
\hline $\begin{array}{l}\text { BRAF, NRAS, KIT, GNAQ, CDK4, PTK2B, ERBB4, } \\
\text { GNA11, MEK, MITF, AKT3, MMP, CXCR4, EPHA3, } \\
\text { FAS, PIK3CA, MET, CTNNB1, NEK10, PDGFRA }\end{array}$ & $\begin{array}{l}\text { Melanoma, asymmetry of skin architecture, } \\
\text { skin pigmentation, change in skin color, size } \\
\text { and diameter, nuclear atypia }\end{array}$ & $\begin{array}{l}\text { Mitochondrial dysfunction, mitochondrial } \\
\text { respiratory complex deficiency, elevated ROS } \\
\text { levels, dysregulated antioxidant defense } \\
\text { system, decreased mitochondrial OXPHOS } \\
\text { and increased Warburg effect. }\end{array}$ & $70,147,148$ \\
\hline PTCH1; PTCH2 & $\begin{array}{l}\text { Basal cell carcinoma, erythematous patch, } \\
\text { papule, nodule or plaque which is often } \\
\text { eroded, ulcerated or indurated, }\end{array}$ & $\begin{array}{l}\text { Mitochondrial dysfunction, mitochondrial } \\
\text { respiratory complex deficiency, elevated ROS } \\
\text { levels, dysregulated antioxidant defense } \\
\text { system, decreased mitochondrial OXPHOS, } \\
\text { altered metabolism. }\end{array}$ & 149 \\
\hline
\end{tabular}

Skin manifestations of congenital mitochondriopathies

Although congenital mitochondrial diseases are primarily neuro-musculopathies, various organs are dependent on mitochondrial respiration and therefore affected by organelle dysfunction. Several reports have surfaced over the years regarding the cutaneous involvement of mitochondrial syndromes, although the results are heterogeneous and lack consensus. Bodemer et al. ${ }^{88}$ observed 14 children with assorted mitochondria-related disorders (e.g., myopathies, Leigh syndrome, deafness, Wolfram syndrome, hypotonia, Pearson syndrome, etc.), and detected specific skin and hair abnormalities like alopecia, brittle hair, rashes, mottled pigmentation, erythematous lesions, acrocyanosis, hypertrichosis, etc. Abnormal OXPHOS was confirmed using cultured skin fibroblasts from all patients, with abnormal ETC protein expression in 3 cases and mtDNA aberrations in one case. The authors surmised that a mitochondriopathy should be considered if sudden and unexplained cutaneous signs manifest along with an unrelated disorder. Sonam et al. ${ }^{89}$ assessed 25 children with Leigh syndrome, a neurometabolic disorder involving both mitochondrial and nuclear mutations, and observed hypertrichosis and hypopigmentation in most patients. Two mtDNA mutations-T3271C and A3243G-in the MELAS syndrome are associated with nail disorders ${ }^{90}$. Hypomelanosis has also been observed in some cases of Kearns-Sayre syndrome, a multi-organ mitochondrial disorder characterized by large mtDNA deletions ${ }^{91}$. Alper's syndrome is a rare, autosomal recessive neurological disorder caused by mutation(s) in the POLG1 gene. Campuzano-García et al. ${ }^{92}$ reported a case of a 10-month-old boy with Alpers syndrome in Mexico (2015) that showed hyperpigmented macules with hypochromic centers in the skin folds of the armpits, groin, and foot dorsum. The rashes were bilateral and mostly concentrated over the dorsal regions. AlSaman et al. ${ }^{93}$ reported a case of a hypotonic 12-month old infant in Saudi Arabia with mtDNA depletion syndrome (MDS) who also showed sparse and brittle hair, scaly skin, eczema, and reticular pigmentation.

The Hutchinson-Gilford progeria syndrome (HGPS) is a very rare congenital disease caused by a dominant mutation in the lamin A gene, which encodes for a structural protein that maintains mitochondrial shape. The cutaneous symptoms of HGPS include hair loss (alopecia), extensive skin wrinkling, and loss of subcutaneous fat that further lends an aged appearance. A recent study on a progeroid patient in Bangladesh reported novel maternally inherited mtDNA mutations, especially in the D-loop region ${ }^{94}$, which correlates with reduced OXPHOS and mitochondrial dysfunction seen in dermal fibroblasts isolated from HGPS patients ${ }^{95}$.

\section{Skin diseases caused by mitochondrial dysfunction}

Several skin diseases are associated with mutations in mitochondrial proteins encoded by mitochondrial or nuclear genes or other aberrations in mtDNA that lead to disrupted OXPHOS, excessive ROS generation, mitochondrial fragmentation, which directly results in the cutaneous symptoms. For instance, non-epidermolytic palmoplantar keratoderma (NEPPK) is associated with the A7445G mutation in mtDNA, which affects the ETC complex IV subunit 1, and decreases cellular respiration $^{96}$. Dupuytren's disease, a maternally inherited condition characterized by abnormal thickening of the skin of the hands, is caused by a mutation in mtDNA $16 \mathrm{~S}$ 
rRNA $^{97}$. The molecular basis of several skin diseases is reduced mitochondrial respiration due to mutations in the nuclear genes encoding OXPHOS proteins. For instance, mutations in the complex IV-encoding nuclear gene $C O X 7 B$ is responsible for the rare autosomal dominant aplasia cutis congenita (APLCC), which is typified by the congenital absence of the epidermis ${ }^{98}$. Besides, a defective complex III due to mutated $\mathrm{BC} 1$ (ubiquinol-cytochrome c reductase) synthesis-like protein is the genetic basis of the Bjornstad syndrome, an autosomal recessive disease characterized by twisted hair shaft and brittle hair ${ }^{99}$. The iron-porphyrin complex heme is essential for cytochrome c function, and the heme synthesis pathway involves both nuclear and mitochondrial proteins. Mutations in genes encoding for heme biosynthesis enzymes often result in photosensitivity and skin inflammatory conditions like dermatitis ${ }^{100}$. The Krebs cycle provides essential metabolites for heme synthesis, and its disruption has been linked with alopecia and pigmented rashes. Epidermolysis bullosa simplex (EBS), a unique skin condition characterized by recurrent epidermal blistering following minor abrasions or bruises, is caused by mutations in the Plec1 gene that encodes for the outer mitochondrial membrane protein plectin. Plec1 mutations not only result in abnormally shaped mitochondria but also complex I and complex IV inhibition ${ }^{101}$. Mutations in the mitochondrial pyrroline-5-carboxylate reductase 1 protein result in extensive fragmentation of the mitochondrial network, which is the genetic basis of autosomal recessive cutis laxa type IIB (ARCL2B), a progeroid skin syndrome characterized by wrinkled and sagging skin ${ }^{102}$.

\section{Skin manifestations of genetic diseases affecting the mitochondria}

Mutations in DNA repair genes indirectly lead to mitochondrial dysfunction due to the accumulation of defective mtDNA and form the genetic basis of multiple congenital disorders with skin involvement. For instance, the Rothmund-Thomson syndrome, which presents cutaneous symptoms like sparse eyebrows and eyelashes, is caused by a mutated helicase RecQ protein-like 4 (RECQL4). The absence of RECQL4 is associated with the accumulation of mtDNA damage and a compensatory increase in mtDNA copy number ${ }^{103}$. Mutations in the RECQL2 ligase are known to increase mitochondrial ROS production and are the cause of Werner syndrome, a rare autosomal recessive adult progeria with considerable skin involvement ${ }^{104}$. The genetic basis of the neurodegenerative disorder ataxia-telangiectasia (AT) is the loss of the AT mutated $(A T M)$ gene, which is the master regulator of the DNA damage response. There is significant heterogeneity in its symptoms, and some affected individuals show premature graying of hair, vitiligo, blotchy pigmentation, and inflamed granulomatous lesions. Lack of the ATM gene not only causes massive genomic instability but also impairs mtDNA repair, resulting in reduced OXPHOS and increased mitophagy ${ }^{105}$. There is strong evidence linking aging-associated telomere shortening and impaired mitochondrial metabolism. Dyskeratosis congenita $(\mathrm{DKC})$ is a progressive dermatological condition characterized by premature graying and wrinkling, nail dystrophy, and abnormal pigmentation, and caused by mutations in the telomerase RNA component (TERC) and telomerase reverse transcriptase (TERT) genes ${ }^{106,107}$. Although there is no direct evidence, the significant increase in oxidative stress in cells harboring the above mutations indicate mitochondrial involvement. In addition to impaired mtDNA repair, autoantibodies against mitochondrial proteins (mtABs) also result in mitochondrial dysfunction. High levels of mtABs against multiple mitochondrial proteins have been detected in autoimmune skin disorders like systemic lupus erythematosus (SLE) and pemphigus vulgaris ${ }^{108,109}$. Consistent with this, keratinocytes isolated from pemphigus vulgaris patients show increased ROS levels and impaired OXPHOS ${ }^{110}$.

\section{Mitochondrial targeting for skin regeneration}

Therapeutic targeting of mitochondria in the skin involves either boosting ATP production or scavenging the excess amounts of free radicals. For instance, numerous studies have demonstrated the anti-aging effects of CoQ10 on cultured human dermal fibroblasts $^{44}$. It is a critical ingredient in many anti-aging and regenerative skin creams, and as shown by Knott et al. ${ }^{111}$ topical application of two different CoQ10-containing formulas significantly replenished the levels of this antioxidant in the dermal and epidermal layers of older skin (>60 years). Photo-stressed skin benefitted from CoQ10 application on account of reduction in in situ free radical levels. Schniertshauer et al. ${ }^{112}$ further showed that CoQ10 could restore ATP production, prevent mitophagy, and alleviate oxidative stress in aged skin cells. Nicotinamide, also known as Vitamin B3 or niacinamide, is the precursor of NAD+, which is required for mitochondrial ATP production. The deficiency of nicotinamide causes pellagra, which is characterized by photosensitive dermatitis. The topical application of nicotinamide has shown antiinflammatory effects against rosacea, acne, autoimmune skin conditions, and hyperpigmentation. It is a popular additive in "high-end" formulations for regenerating aged and sun-damaged $\operatorname{skin}^{113}$. Vitamin $\mathrm{C}$ or ascorbic acid is a potent antioxidant that is used in various anti-wrinkle creams to increase collagen synthesis, and topical application of 10\% Vitamin C reversed UV-induced oxidative damage by scavenging $\operatorname{ROS}^{114}$. There is evidence that resveratrol directly improves mitochondrial function in 
various cell types by increasing organelle biogenesis and attenuating ROS production ${ }^{115}$. In addition to cosmetic problems, the treatment of inflammatory and other serious skin diseases also involves mitochondrial targeting. For instance, the anti-inflammatory glucocorticoids that are used to treat SLE and pemphigus vulgaris increase mitochondrial membrane potential at low doses but reduce mitochondrial function at high doses ${ }^{116}$. Taken together, bioactive compounds targeting the mitochondria have proved effective against age-related as well as UV-induced skin damage, in addition to different skin diseases with mitochondrial involvement.

\section{Acknowledgements}

We thank Yuva Biosciences team members for their support. K.K.S. is supported by an NIH grant R01CA204430. We apologize to authors whose work we could not cite due to space limitations.

\section{Author details}

${ }^{1}$ Yuva Biosciences, 1500 1st Avenue N, Birmingham, AL 35203, USA. ${ }^{2}$ Department of Genetics, University of Alabama at Birmingham, Birmingham, AL 35294, USA. ${ }^{3}$ Integartive Center For Aging Research and O'Neal Comprehensive Cancer Center, University of Alabama at Birmingham, Birmingham, AL 35294, USA

\section{Conflict of Interest}

K.K.S. is the scientific founder and chief scientific advisor at Yuva Biosciences. A. S., L.A.-A., and K.K.S. hold equity in Yuva Biosciences.

\section{Publisher's note}

Springer Nature remains neutral with regard to jurisdictional claims in published maps and institutional affiliations.

Received: 30 March 2020 Revised: 22 May 2020 Accepted: 25 May 2020 Published online: 09 June 2020

\section{References}

1. Rittié, L. \& Fisher, G. J. Natural and sun-induced aging of human skin. Cold Spring Harb. Perspect. Med. 5, 1 (2015).

2. Fisher, G. J. et al. Mechanisms of photoaging and chronological skin aging Arch. Dermatol. 138, 1462-1470 (2002).

3. D'Orazio, J., Jarrett, S., Amaro-Ortiz, A. \& Scott, T. UV radiation and the skin. Int. J. Mol. Sci. 14, 12222-12248 (2013).

4. Cooper, G. M. The Cell: A Molecular Approach. The Mechanism of Oxidative Phosphorylation 2nd edn, (Sinauer Associates, Sunderland, MA, 2000).

5. Detmer, S. A. \& Chan, D. C. Functions and dysfunctions of mitochondrial dynamics. Nat. Rev. Mol. Cell Biol. 8, 870-879 (2007).

6. Murphy, M. P. How mitochondria produce reactive oxygen species. Biochem. J. 417, 1-13 (2009)

7. Kirkinezos, I. G. \& Moraes, C. T. Reactive oxygen species and mitochondrial diseases. Semin Cell Dev. Biol. 12, 449-57 (2001).

8. Taanman, J. W. The mitochondrial genome: structure, transcription, translation and replication. Biochim. Biophys. Acta 1410, 103-23 (1999).

9. Neiman, M. \& Taylor, D. R. The causes of mutation accumulation in mitochondrial genomes. Proc. Biol. Sci. 276, 1201-1209 (2009).

10. Taylor, R. W. \& Turnbull, D. M. Mitochondrial DNA mutations in human disease. Nat. Rev. Genet. 6, 389-402 (2005).

11. Bussard, K. M. \& Siracusa, L. D. Understanding Mitochondrial Polymorphisms in Cancer. Cancer Res. 77, 6051-6059 (2017).

12. Adams, J. C. \& Watt, F. M. Changes in keratinocyte adhesion during terminal differentiation: reduction in fibronectin binding precedes alpha 5 beta 1 integrin loss from the cell surface. Cell 63, 425-435 (1990).
13. Lisowski, P., Kannan, P., Mlody, B. \& Prigione, A. Mitochondria and the dynamic control of stem cell homeostasis. EMBO Rep. 19, 45432 (2018).

14. Hamanaka, R. B. et al. Mitochondrial reactive oxygen species promote epidermal differentiation and hair follicle development. Sci. Signal. 6, 261 (2013).

15. Elias, P., Ahn, S., Brown, B., Crumrine, D. \& Feingold, K. R. Origin of the epidermal calcium gradient: regulation by barrier status and role of active vs passive mechanisms. J. Invest. Dermatol. 119, 1269-74 (2002).

16. Pillai, S., Bikle, D. D., Mancianti, M. L., Cline, P. \& Hincenbergs, M. Calcium regulation of growth and differentiation of normal human keratinocytes: modulation of differentiation competence by stages of growth and extracellular calcium. J. Cell Physiol. 143, 294-302 (1990).

17. Bhaduri, A. et al. Network analysis identifies mitochondrial regulation of epidermal differentiation by MPZL3 and FDXR. Dev. Cell. 35, 444-57 (2015).

18. Allombert-Blaise, $C$. et al. Terminal differentiation of human epidermal keratinocytes involves mitochondria- and caspase-dependent cell death pathway. Cell Death Differ. 10, 850-852 (2003).

19. Slominski, A. T. et al. Melatonin, mitochondria, and the skin. Cell Mol. Life Sci. 74, 3913-3925 (2017).

20. Slominski, R. M., Reiter, R. J., Schlabritz-Loutsevitch, N., Ostrom, R. S. \& Slominski, A. T. Melatonin membrane receptors in peripheral tissues: distribution and functions. Mol. Cell Endocrinol. 351, 152-166 (2012).

21. Kleszczynski, K., Tukaj, S., Kruse, N., Zillikens, D. \& Fischer, T. W. Melatonin prevents ultraviolet radiation-induced alterations in plasma membrane potential and intracellular pH in human keratinocytes. J. Pineal Res. 54, 89-99 (2013).

22. Fischer, T. W., Kleszczynski, K., Hardkop, L. H., Kruse, N. \& Zillikens, D. Melatonin enhances antioxidative enzyme gene expression (CAT, GPx, SOD), prevents their UVR-induced depletion, and protects against the formation of DNA damage (8-hydroxy-2'-deoxyguanosine) in ex-vivo human skin. J. Pineal Res. 54, 303-312 (2013).

23. Kilanczyk, E. \& Bryszewska, M. The effect of melatonin on antioxidant enzymes in human diabetic skin fibroblasts. Cell Mol. Biol. Lett. 8, 333-336 (2003).

24. Reiter, R. J. et al. Melatonin Mitigates Mitochondrial Meltdown: Interactions with SIRT3. Int. J. Mol. Sci. 19, 2439 (2018).

25. He, C. et al. Mitochondria synthesize melatonin to ameliorate its function and improve mice oocyte's quality under in-vitro conditions. Int. J. Mol. Sci. 17, 939 (2016).

26. Reiter, R. J. et al. Mitochondria: central organelles for melatonin's antioxidant and anti-aging actions. Molecules 23, 509 (2018).

27. Fernández, A., Ordóñez, R., Reiter, R. J., González-Gallego, J. \& Mauriz, J. L. Melatonin and endoplasmic reticulum stress: relation to autophagy and apoptosis. J. Pineal Res. 59, 292-307 (2015)

28. Kim, T. K. et al. Metabolism of melatonin and biological activity of intermediates of melatoninergic pathway in human skin cells. FASEB J. 27, 2742-55 (2013).

29. Romić, M. D. et al. Melatonin-loaded chitosan/Pluronic ${ }^{\circledR}$ F127 microspheres as in situ forming hydrogel: an innovative antimicrobial wound dressing. Eur. J. Pharm. Biopharm. 107, 67-79 (2016).

30. Kimmel, H. M., Grant, A. \& Ditata, J. The presence of oxygen in wound healing. Wounds 28, 264-70 (2016)

31. Krutmann, J., \& Gilchrest, B. A. Photoaging of skin. In: Skin Aging (eds Gilchrest, B. A. \& Krutmann, J.) 33-43. (Springer, New York 2006).

32. Krutmann, J. \& Schroeder, P. Role of mitochondria in photoaging of human skin: the defective powerhouse model. J. Investigative Dermatol. Symp. Proc 14, 44-49 (2009).

33. Richter, C. Oxidative damage to mitochondrial DNA and its relationship to ageing. Int. J. Biochem. Cell Biol. 27, 647-53 (1995).

34. Yang, J. H., Lee, H. C. \& Wei, Y. H. Photoageing-associated mitochondrial DNA length mutations in human skin. Arch. Dermatol. Res. 287, 641-8 (1995).

35. Kaneko, N. et al. Mitochondrial common deletion mutation and extrinsic skin ageing in German and Japanese women. Exp. Dermatol. 21, 26-30 (2012).

36. Singh, B., Schoeb, T. R., Bajpai, P., Slominski, A. \& Singh, K. K. Reversing wrinkled skin and hair loss in mice by restoring mitochondrial function. Cell Death Dis. 9, 735 (2018).

37. Prahl, S. et al. Aging skin is functionally anaerobic: importance of coenzyme Q10 for anti-aging skin care. Biofactors 32, 245-55 (2008).

38. Bowman, A. \& Birch-Machin, M. A. Age-dependent decrease of mitochondrial complex II activity in human skin fibroblasts. J. Invest. Dermatol. 136, 912-919 (2016). 
39. Lemasters, J. J. Selective mitochondrial autophagy, or mitophagy, as a targeted defense against oxidative stress, mitochondrial dysfunction, and aging Rejuvenation Res. 8, 3-5 (2005).

40. Aymard, E. et al. Autophagy in human keratinocytes: an early step of the differentiation? Exp. Dermatol. 20, 263-8 (2011).

41. Mellem, D. et al. Fragmentation of the mitochondrial network in skin in vivo. PLoS One 12, 1462-1470 (2017).

42. Jugé, R. et al. Quantification and characterization of UVB-induced mitochondrial fragmentation in normal primary human keratinocytes. Sci. Rep. $\mathbf{6}$, 35065 (2016).

43. Bentinger, M., Tekle, M. \& Dallner, G. Coenzyme Q-biosynthesis and functions. Biochem. Biophys. Res. Commun. 396, 74-79 (2010).

44. Hoppe. et al. Coenzyme Q10, a cutaneous antioxidant and energizer. Biofactors 9, 371-378 (1999).

45. Blatt, T. \& Littarru, G. P. Biochemical rationale and experimental data on the antiaging properties of $\mathrm{CoQ}(10)$ at skin level. Biofactors 37, 381-385 (2011).

46. Marcheggiani, F. et al. Modulation of coenzyme Q(10) content and oxidative status in human dermal fibroblasts using HMG-CoA reductase inhibitor over a broad range of concentrations. From mitohormesis to mitochondrial dysfunction and accelerated aging. Aging 11, 2565-2582 (2019).

47. Fisher, G. J. et al. Pathophysiology of premature skin aging induced by ultraviolet light. N. Engl. J. Med. 337, 1419-28 (1997).

48. Berneburg, M. et al. Chronically ultraviolet-exposed human skin shows a higher mutation frequency of mitochondrial DNA as compared to unexposed skin and the hematopoietic system. Photochem. Photobiol. 66, 271-5 (1997).

49. Berneburg, M. et al. Induction of the photoaging-associated mitochondrial common deletion in vivo in normal human skin. J. Invest. Dermatol. 122, 1277-83 (2004).

50. Krishnan, K. J., Harbottle, A. \& Birch-Machin, M. A. The use of a 3895 bp mitochondrial DNA deletion as a marker for sunlight exposure in human skin. J. Invest. Dermatol. 123, 1020-4 (2005).

51. Powers, J. M., Murphy, G., Ralph, N., O'Gorman, S. M., Murphy, J. E. \& Mitochondrial, D. N. A. deletion percentage in sun exposed and non-sun exposed skin. J. Photochem. Photobiol. B 165, 277-82 (2016).

52. Berneburg, M. \& Lehmann, A. R. Xeroderma pigmentosum and related disorders: defects in DNA repair and transcription. Adv. Genet. 43, 71-102 (2001).

53. Berneburg, M. \& Krutmann, J. Photoaging-associated large-scale deletions of mitochondrial DNA. Methods Enzymol. 319, 366-76 (2000).

54. Balaban, R. S., Nemoto, S. \& Finkel, T. Mitochondria, oxidants, and aging. Cell 25, 483-95 (2005).

55. Schroeder, P., Gremmel, T., Berneburg, M. \& Krutmann, J. Partial depletion of mitochondrial DNA from human skin fibroblasts induces a gene expression profile reminiscent of photoaged skin. J. Invest. Dermatol. 128, 2297-303 (2008)

56. Majora, M. et al. Functional consequences of mitochondrial DNA deletions in human skin fibroblasts: increased contractile strength in collagen lattices is due to oxidative stress-induced lysyl oxidase activity. Am. J. Pathol. 175, 1019-29 (2009).

57. Oyewole, A. O., Wilmot, M. C., Fowler, M. \& Birch-Machin, M. A. Comparing the effects of mitochondrial targeted and localized antioxidants with cellular antioxidants in human skin cells exposed to UVA and hydrogen peroxide. FASEB J. 28, 485-94 (2014).

58. Schieke, S. et al. Infrared-A radiation-induced matrix metalloproteinase 1 expression is mediated through extracellular signal-regulated kinase 1/2 activation in human dermal fibroblasts. J. Invest. Dermatol. 119, 1323-9 (2002)

59. Kim, H. H. et al. Augmentation of UV-induced skin wrinkling by infrared irradiation in hairless mice. Mech. Ageing Dev. 126, 1170-7 (2005).

60. Schauen, M., Hornig-Do, H. T., Schomberg, S., Herrmann, G. \& Wiesner, R. J. Mitochondrial electron transport chain activity is not involved in ultraviolet $\mathrm{A}$ (UVA)-induced cell death. Free Radic. Biol. Med. 42, 499-509 (2007).

61. Schroeder, P. et al. Cellular response to infrared radiation involves retrograde mitochondrial signaling. Free Radic. Biol. Med. 43, 128-35 (2007).

62. Karu, T. I., Pyatibrat, L. V. \& Afanasyeva, N. I. A novel mitochondrial signaling pathway activated by visible-to-near infrared radiation. Photochem. Photobiol. 80, 366-72 (2004).

63. Sander, C. S. et al. Photoaging is associated with protein oxidation in human skin in vivo. J. Invest. Dermatol. 118, 618-25 (2002).

64. Ortonne, J. P. Pigmentary changes of the ageing skin. Br. J. Dermatol. 122 21-8 (1990).
65. Hardman, J. A. et al. The peripheral clock regulates human pigmentation. J. Invest. Dermatol. 135, 1053-1064 (2015).

66. Severi, G. \& English, D. J. Prevention of Skin Cancer, Cancer Prevention-Cancer Causes Vol. 3 (eds Hill, D, Elwood, J. M, \& English, D. J) 73-88 (Kluwer Academic Publishers. Dordrecht, 2004).

67. Armstrong, B. K. \& Kricker, A. The epidemiology of UV induced skin cancer. J. Photochem. Photobiol. B 63, 8-18 (2001).

68. Takeuchi, H., Fujimoto, A. \& Hoon, D. S. Detection of mitochondrial DNA alterations in plasma of malignant melanoma patients. Ann. N. Y Acad. Sci. 1022, 50-54 (2004).

69. Mithani, S. K., Smith, I. M., Topalian, S. L. \& Califano, J. A. Nonsynonymous somatic mitochondrial mutations occur in the majority of cutaneous melanomas. Melanoma Res. 18, 214-219 (2008).

70. Deichmann, $M$. et al. Somatic mitochondrial mutations in melanoma resection specimens. Int. J. Oncol. 24, 137-141 (2004).

71. Ebner, S. et al. Mitochondrial haplogroups, control region polymorphisms and malignant melanoma: a study in middle European Caucasians. PLoS One 6, 12 (2011)

72. Zhang, W. et al. Single nucleotide polymorphisms in the mitochondrial displacement loop region modifies malignant melanoma: a study in Chinese Han population. Mitochondrial DNA 26, 205-7 (2015).

73. Durham, S. E., Krishnan, K. J., Betts, J., Birch-Machin, M. A. \& Mitochondrial, D. N. A. damage in non-melanoma skin cancer. Brit J. Cancer 88, 90-95 (2003).

74. Yang, J. H., Lee, H. C., Chung, J. G., Wei, Y. H. \& Mitochondrial, D. N. A mutations in light-associated skin tumors. Anticancer Res. 24, 1753-1758 (2004).

75. Hu, L., Yao, X. \& Shen, Y. Altered mitochondrial DNA copy number contributes to human cancer risk: evidence from an updated meta-analysis. Sci. Rep. 6, 35859 (2016)

76. Robbins, D. \& Zhao, Y. Manganese superoxide dismutase in cancer prevention. Antioxid. Redox Signal. 20, 1628-45 (2014).

77. Zhao, Y. et al. A mechanism-based antioxidant approach for the reduction of skin carcinogenesis. Cancer Res. 65, 1401-5 (2005).

78. Ibarrola-Villava, M. et al. Role of glutathione S-transferases in melanoma susceptibility: association with GSTP1 rs1695 polymorphism. Br. J. Dermatol. 166, 1176-83 (2012).

79. Valverde, P. et al. Melatonin antagonizes alpha-melanocyte-stimulating hormone enhancement of melanogenesis in mouse melanoma cells by blocking the hormone-induced accumulation of the c locus tyrosinase. Eur. J. Biochem. 232, 257-63 (1995).

80. Lissoni, P. et al. A phase II study of chemoneuroimmunotherapy with platinum, subcutaneous low-dose interleukin-2 and the pineal neurohormone melatonin (P.I.M.) as a second-line therapy in metastatic melanoma patients progressing on dacarbazine plus interferon-alpha. In Vivo 16, 93-6 (2002)

81. Ghaderi, R., Sehatbakhsh, S., Bakhshaee, M. \& Sharifzadeh, G. R. Urinary melatonin levels and skin malignancy. Iran J. Med. Sci. 39, 64-67 (2014).

82. Warburg, O. Versuche am überlebenden Karzinomgewebe. Biochem. Z. 142, 317-333 (1923).

83. DeBerardinis, R. J., Lum, J. J., Hatzivassiliou, G. \& Thompson, C. B. The biology of cancer: metabolic reprogramming fuels cell growth and proliferation. Cell Metab. 7, 11-20 (2008)

84. Papandreou, I., Cairns, R. A., Fontana, L., Lim, A. L. \& Denko, N. C. HIF-1 mediates adaptation to hypoxia by actively downregulating mitochondria oxygen consumption. Cell Metab. 3, 187-197 (2006).

85. Hall, A. et al. Dysfunctional oxidative phosphorylation makes malignant melanoma cells addicted to glycolysis driven by the (V600E) BRAF oncogene. Oncotarget 4, 584-599 (2013).

86. Mamelak, A. J. et al. Downregulation of NDUFA1 and other oxidative phosphorylation-related genes is a consistent feature of basal cell carcinoma. Exp. Dermatol. 14, 336-48 (2005).

87. Feichtinger, R. G., Sperl, W., Bauer, J. W. \& Kofler, B. Mitochondrial dysfunction: a neglected component of skin diseases. Exp. Dermatol. 23, 607-14 (2014).

88. Bodemer, C. et al. Hair and skin disorders as signs of mitochondrial disease. Pediatrics 103, 428-33 (1999).

89. Sonam, $\mathrm{K}$ et al. Clinical and magnetic resonance imaging findings in patients with Leigh syndrome and SURF1 mutations. Brain Dev. 36, 807-12 (2014).

90. McPherson, E. \& Zabel, C. Mitochondrial mutation in a child with distal arthrogryposis. Am. J. Med. Genet. A 140, 184-5 (2006).

91. Kakourou, T. et al. Kearns Sayre syndrome initially presenting as hypomelanosis of Ito. Arch. Dis. Child 81, 280-1 (1999). 
92. Campuzano-García, A. E., Rodríguez-Arámbula, A., Torres-Alvarez, B. \& Castanedo-Cázares, J. P. Hyperpigmentation and atrophy in folds as cutaneous manifestation in a case of mitochondrial myopathy. Dermatol. Online J. 21, 5 (2015).

93. AlSaman, A., Tomoum, H., Invernizzi, F. \& Zeviani, M. Hepatocerebral form of mitochondrial DNA depletion syndrome due to mutation in MPV17 gene. Saudi J. Gastroenterol. 18, 285-9 (2012).

94. Rahman, A. et al. Hutchinson-Gilford progeria syndrome (HGPS) with mitochondrial dna (mtDNA) HV1 control region mutations. Unique J. Pharm. Biol. Sci. 2, 1 (2014).

95. Rivera-Torres, J. et al. Identification of mitochondrial dysfunction in Hutchinson-Gilford progeria syndrome through use of stable isotope labeling with amino acids in cell culture. J. Proteom. 91, 466-477 (2013).

96. Maasz, A. et al. Phenotypic variants of the deafness-associated mitochondrial DNA A7445G mutation. Curr. Med. Chem. 15, 1257-1262 (2008).

97. Michou, L. et al. Genetics of Dupuytren's disease. Jt. Bone Spine 79, 7-12 (2012).

98. Indrieri, A. et al. Mutations in COX7B cause microphthalmia with linear skin lesions, an unconventional mitochondrial disease. Am. J. Hum. Genet. 91, 942-9 (2012).

99. Lynn, A. M., King, R. I., Mackay, R. J., Florkowski, C. M. \& Wilson, C. J. BCS1L gene mutation presenting with GRACILE-like syndrome and complex III deficiency. Ann. Clin. Biochem. 49, 201-3 (2012).

100. Murphy, G. M. The cutaneous porphyrias: a review. The British Photodermatology Group. Br. J. Dermatol. 140, 573-81 (1999).

101. Maselli, R. A. et al. Congenital myasthenic syndrome associated with epidermolysis bullosa caused by homozygous mutations in PLEC1 and CHRNE. Clin. Genet. 80, 444-451 (2011).

102. Dimopoulou, A. et al. Genotype-phenotype spectrum of PYCR1-related autosomal recessive cutis laxa. Mol. Genet. Metab. 110, 352-361 (2013).

103. Kazak, L., Reyes, A. \& Holt, I. J. Minimizing the damage: repair pathways keep mitochondrial DNA intact. Nat. Rev. Mol. Cell Biol. 13, 659-71 (2012).

104. Labbé, A. et al. The Werner syndrome gene product (WRN): a repressor of hypoxia-inducible factor-1 activity. Exp. Cell Res. 318, 1620-32 (2012).

105. Sharma, N. K. et al. Intrinsic mitochondrial DNA repair defects in Ataxia Telangiectasia. DNA Repair 13, 22-31 (2014).

106. Sahin, E. et al. Telomere dysfunction induces metabolic and mitochondrial compromise. Nature 470, 359-65 (2011).

107. Sharma, N. K. et al. Human telomerase acts as a hTR-independent reverse transcriptase in mitochondria. Nucleic Acids Res. 40, 712-25 (2012).

108. Perl, A., Gergely, P. Jr. \& Banki, K. Mitochondrial dysfunction in T cells of patients with systemic lupus erythematosus. Int Rev. Immunol. 23, 293-313 (2004)

109. Di Zenzo, G., Zambruno, G. \& Borradori, L. Endemic pemphigus foliaceus: towards understanding autoimmune mechanisms of disease development. J. Invest. Dermatol. 132, 2499-502 (2012).

110. Kalantari-Dehaghi, M. et al. Mechanisms of mitochondrial damage in keratinocytes by pemphigus vulgaris antibodies. J. Biol. Chem. 288, 16916-16925 (2013)

111. Knott, A. et al. Topical treatment with coenzyme Q10-containing formulas improves skin's Q10 level and provides antioxidative effects. Biofactors 41, 383-90 (2015).

112. Schniertshauer, D. et al. Accelerated Regeneration of ATP Level after Irradiation in Human Skin Fibroblasts by Coenzyme Q10. Photochem. Photobiol. 92, 488-94 (2016).

113. Bains, P., Kaur, M., Kaur, J. \& Sharma, S. Nicotinamide: Mechanism of action and indications in dermatology. Indian J. Dermatol. Venereol. Leprol. 84 234-237 (2018)

114. Telang, P. S. Vitamin C in dermatology. Indian Dermatol. Online J. 4(2), 143-6 (2013)

115. Ungvari, Z., Sonntag, W. E., de Cabo, R., Baur, J. A. \& Csiszar, A. Mitochondria protection by resveratrol. Exerc Sport Sci. Rev. 39, 128-32 (2011).

116. Du, J. et al. Flow cytometry analysis of glucocorticoid receptor expression and binding in steroid-sensitive and steroid-resistant patients with systemic lupus erythematosus. Arthritis Res. Ther. 11, R108 (2009).

117. Valentin-Vega, Y. A. \& Kastan, M. B. A new role for ATM: regulating mitochondrial function and mitophagy. Autophagy 8, 840-841 (2012).

118. Pallardó, F. V. et al. Mitochondrial dysfunction in some oxidative stress-related genetic diseases: Ataxia-Telangiectasia, Down Syndrome, Fanconi Anaemia and Werner Syndrome. Biogerontology 11, 401-419 (2010).
119. Hymes, J., Stanley, C. M. \& Wolf, B. Mutations in BTD causing biotinidase deficiency. Hum. Mutat. 18, 375-381 (2001).

120. Hernandez-Vazquez, A. et al. Biotinidase knockout mice show cellular energy deficit and altered carbon metabolism gene expression similar to that of nutritional biotin deprivation: clues for the pathogenesis in the human inherited disorder. Mol. Genet. Metab. 110, 248-254 (2013).

121. Pedespan, J. M. et al. Menkes disease: study of the mitochondrial respiratory chain in three cases. Eur. J. Paediatr. Neurol. 3, 167-170 (1999).

122. Atamna, H., Newberry, J., Erlitzki, R., Schultz, C. S. \& Ames, B. N. Biotin deficiency inhibits heme synthesis and impairs mitochondria in human lung fibroblasts. J. Nutr. 137, 25-30 (2007).

123. Hinson, J. T. et al. Missense mutations in the BCS1L gene as a cause of the Björnstad syndrome. N. Engl. J. Med. 356, 809-819 (2007).

124. Selvaag, E. Pili torti et canaliculi and agenesis of the teeth: report of a new "pure" hair-tooth ectodermal dysplasia in a Norwegian family. J. Med. Genet. 37, 721-723 (2000)

125. Finsterer, J. \& Zarrouk-Mahjoub, S. Cardiac manifestations of mitochondrial disorders. Tex. Heart Inst. J. 40, 634-635 (2013).

126. Gronow, S., Noah, C., Blumenthal, A., Lindner, B. \& Brade, H. Construction of a deep-rough mutant of Burkholderia cepacia ATCC 25416 and characterization of its chemical and biological properties. J. Biol. Chem. 278, 1647-1655 (2003).

127. Lange, H., Mühlenhoff, U., Denzel, M., Kispal, G. \& Lill, R. The heme synthesis defect of mutants impaired in mitochondrial iron-sulfur protein biogenesis is caused by reversible inhibition of ferrochelatase. J. Biol. Chem. 279, 29101-8 (2004).

128. Bareth, B. et al. The heme a synthase Cox15 associates with cytochrome c oxidase assembly intermediates during Cox1 maturation. Mol. Cell Biol. 33, 4128-4137 (2013).

129. Nilsson, R. et al. Discovery of genes essential for heme biosynthesis through large-scale gene expression analysis. Cell Metab. 10, 119-130 (2009).

130. Zierler, K. A. et al. Functional cardiac lipolysis in mice critically depends on comparative gene identification-58. J. Biol. Chem. 288, 9892-9904 (2013).

131. Scheibye-Knudsen, M., Croteau, D. L. \& Bohr, V. A. Mitochondrial deficiency in Cockayne syndrome. Mech. Ageing Dev. 134, 275-283 (2013).

132. Scheibye-Knudsen, M. P. et al. Cockayne syndrome group B protein prevents the accumulation of damaged mitochondria by promoting mitochondrial autophagy. J. Exp. Med. 209, 855-869 (2012).

133. Ravera, S. et al. Mitochondrial respiratory chain Complex I defects in Fanconi anemia complementation group A. Biochimie 95, 1828-1837 (2013).

134. Cappelli, E. et al. Mitochondrial respiratory complex I defects in Fanconi anemia. Trends Mol. Med. 19, 513-514 (2013).

135. Kleefstra, T. et al. Mitochondrial dysfunction and organic aciduria in five patients carrying mutations in the Ras-MAPK pathway. Eur. J. Hum. Genet. 19 138-144 (2011).

136. Aeby, A et al. Cardiofaciocutaneous (CFC) syndrome associated with muscular coenzyme Q10 deficiency. J. Inherit. Metab. Dis. 30, 827 (2007).

137. Champion, K. J. et al. Germline mutation in BRAF codon 600 is compatible with human development: de novo p.V600G mutation identified in a patient with CFC syndrome. Clin. Genet. 79, 468-474 (2011).

138. Amary, M. F. et al. Ollier disease and Maffucci syndrome are caused by somatic mosaic mutations of IDH1 and IDH2. Nat. Genet. 43, 1262-1265 (2011).

139. Kodama, H., Okabe, I., Yanagisawa, M. \& Kodama, Y. Copper deficiency in the mitochondria of cultured skin fibroblasts from patients with Menkes syndrome. J. Inherit. Metab. Dis. 12, 386-389 (1989).

140. Schroder, R. et al. Disorganization of the desmin cytoskeleton and mitochondrial dysfunction in plectin-related epidermolysis bullosa simplex with muscular dystrophy. J. Neuropathol. Exp. Neurol. 61, 520-530 (2002).

141. Banwell, B. L. et al. Myopathy, myasthenic syndrome, and epidermolysis bullosa simplex due to plectin deficiency. J. Neuropathol. Exp. Neurol. 58, 832-846 (1999).

142. Reversade, B. et al. Mutations in PYCR1 cause cutis laxa with progeroid features. Nat. Genet. 41, 1016-1021 (2009).

143. Croteau, D. L. et al. RECQL4 localizes to mitochondria and preserves mitochondrial DNA integrity. Aging Cell 11, 456-466 (2012).

144. Hermanns, P. et al. RMRP mutations in cartilage-hair hypoplasia. Am. J. Med. Genet A 140, 2121-2130 (2006).

145. Robertson, N. J. et al. Brain lactic alkalosis in Aicardi-Goutières syndrome Neuropediatrics 35, 20-26 (2004) 
146. Wu, L. SAMHD1 knockout mice: modeling retrovirus restriction in vivo. Retrovirology 10, 142 (2013).

147. Larsson, N. G., Tulinius, M. H., Holme, E. \& Oldfors, A. Pathogenetic aspects of the A8344G mutation of mitochondrial DNA associated with MERRF syndrome and multiple symmetric lipomas. Muscle Nerve 18, 102-106 (1995).
148. Dutton-Regester, K. et al. A high-throughput panel for identifying clinically relevant mutation profiles in melanoma. Mol. Cancer Ther. 11, 888-897 (2012).

149. Kamenisch, Y. et al. The mitochondrial DNA common deletion is present in most basal and squamous cell carcinoma samples isolated by laser capture microdissection but generally at reduced rather than increased levels. J. Invest Dermatol. 127, 486-490 (2007). 